\title{
Développement et déclin des chemins de fer dans le Wisconsin
}

Maurice Ed. Perret

Le Wisconsin fait partie de la Nouvelle-France découverte en 1634 par Jean Nicolet. Les premiers blancs qui visitèrent le pays furent de explorateurs, des trappeurs et des missionnaires. En 1669, une mission fut établie dans la vallée du Fox, premier établissement permanent qui, par la suite, prit le nom de De Pere. Les Anglais se rendirent maîtres de la contrée en 1763, mais la cédèrent aux EtatsUnis vingt ans plus tard. Compris d'abord dans le Territoire du Nord-Ouest, le Wisconsin devint un territoire distinct en 1836 et fut admis comme trentième Etat de l'Union en 1848. Jusqu'au début du $\mathrm{XIX}^{\mathrm{c}}$ siècle, la population avait été peu nombreuse. Milwaukee fut établie en 1818 , à l'endroit où se trouvait un village indien, au bord du lac Michigan. En 1819 débuta l'exploitation active de gisements de minerais de plomb, dans le sud-est de l'Etat; ces mines attirèrent d'abord des Américains, puis des mineurs de Cornouaille. En 1840, Milwaukee comptait 1712 habitants et le Wisconsin entier 30 945. Au recensement suivant, en 1850, l'augmentation est très sensible, le nouvel Etat a 305391 habitants, la ville de Milwaukee 20 061, tandis que Madison, la capitale, n'est encore qu'un village de 1525 âmes. L'immigration a été forte et a été constituée principalement par des Allemands. La densité est encore faible, le Wisconsin ayant une superficie de $54705 \mathrm{~km}^{2}$, soit environ trois fois la Suisse.

Aux Etats-Unis, les premières lignes de chemin de fer à vapeur avaient été inaugurées en 1830, et peu à peu le réseau s'était développé surtout dans l'est et le sud-est. En 1850, les transports par bateau restent encore importants et, dans le Wisconsin, les premières voies ferrées sont tracées de manière à relier les voies navigables. La première ligne de chemin de fer de l'Etat est inaugurée en 1850; partant de Milwaukee, elle ne couvre qu'une distance de $16 \mathrm{~km}$ jusqu'à Wauwatosa, un village à cette époque, mais elle marque le début d'un projet ambitieux, la liaison entre le lac Michigan et le Mississippi et la compagnie qui l'a créée, formée sous le nom de «Milwaukee \& Waukesha Railroad Company» a adopté le titre de "Milwaukee and Mississippi Railroad Company». A cette époque, Chicago même, qui par la suite deviendra le centre des chemins de fer du continent, n'a encore qu'une seule ligne qui, en 1850 , s'arrête à Elgin, à 20 km.

Dès l'origine et jusqu'à nos jours, les chemins de fer américains ont été créés par l'initiative privée et restent des entreprises privées. Les compagnies ont évidemment l'intention de servir l'intérêt public, mais les bénéfices sont au premier plan et les lignes ne sont construites que là où les profits semblent assurés. Les recettes proviennent des transports de marchandises, de voyageurs et de poste; elles seront en rapport direct avec la population et l'importance des localités. C'est pourquoi les compagnies chercheront d'abord à relier les grandes villes entre elles, puis les grandes villes avec les villes moyennes, les villes moyennes entre elles et enfin d'autres localités avec les villes. Il n'y a pas de compagnie qui projette de ligne aboutissant dans une région peu peuplée, dans l'espoir que cette dernière se développera, comme c'est le cas dans d'autres pays d'Amérique, par contre les contrées dont la population est déjà appréciable se développeront plus rapidement quand une ligne de chemin de fer les traversera. Les projets des compagnies sont ambitieux, car il faut attirer des capitaux, mais les réalisations sont généralement limitées: certaines compagnies n'arrivent qu'à exploiter quelques kilomètres de voies, comme la "Chicago and Lake Superior Railway Company", dont la ligne relie Cambridge (Wisconsin) à London (Wisconsin), distance de $5 \mathrm{~km}$. D'autres compagnies ne réussissent pas même à construire une ligne. Dans tout le pays, les changements, les fusions, les réorganisations, les faillites de compagnies sont nombreux, et le Wisconsin ne fait pas exception.

Les progrès des chemins de fer dans cet Etat sont d'abord lents: en 1851, la première ligne atteint Waukesha, le réseau entier n'est encore que de $57 \mathrm{~km}$. Il passe à $115 \mathrm{~km}$ en 1852,145 en 1853, 244 en 1854, année où la capitale, Madison, est reliée à Milwaukee, $512 \mathrm{~km}$ en 1855 et 816 en 1856 , lorsque la ligne de Milwaukee à Prairie du Chien, au bord du Mississippi est achevée. D'autres compagnies se sont formées dans le but d'établir de nouvelles lignes ferroviaires, mais rapidement deux compagnies se développent en étendant leur réseau et en rachetant de petites lignes, ce sont la "Milwaukee, Saint Paul Railway Company» qui a succédé à la "Milwaukee and Mississippi Railroad Company» et la "Chicago and North Western Company». La concurrence n'est pas encore aigüe dans le Wisconsin, il y a encore beaucoup d'espace et les lignes ne se doublent pas. En 1871, lors de la publication du premier indicateur général des che- 


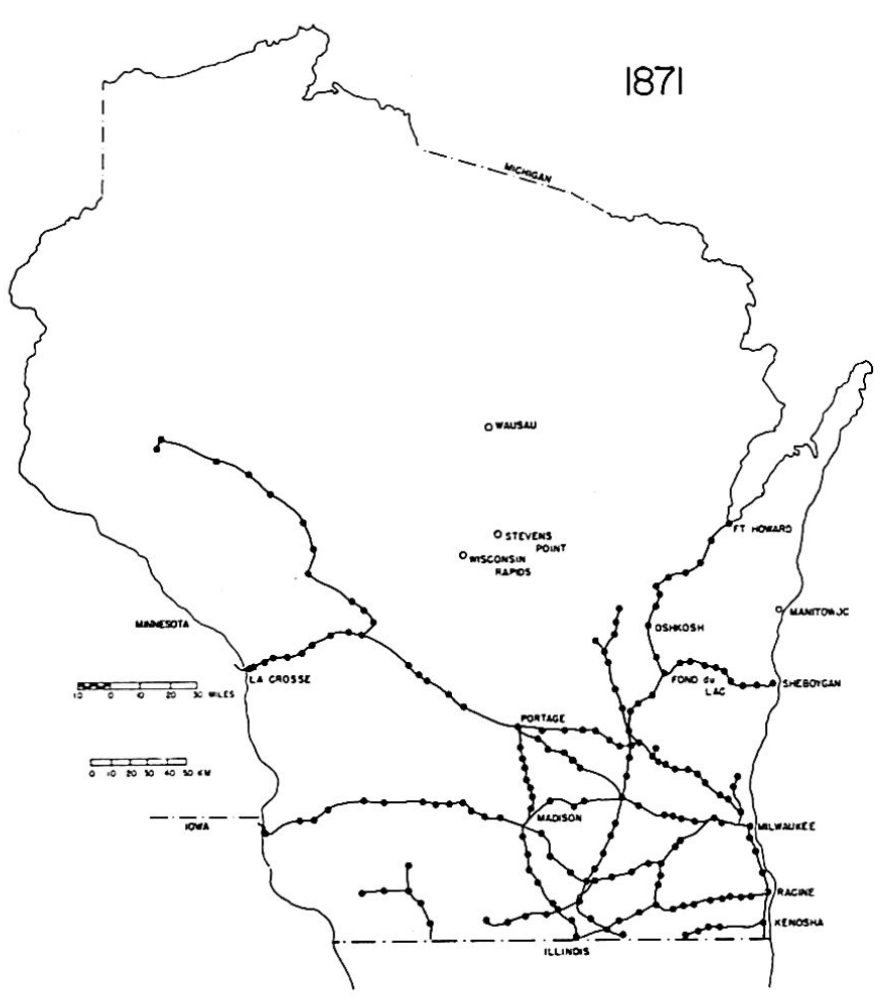

Figure 1. Lignes avec services de voyageurs en 1871

mins de fer des Etats-Unis, sept compagnies exploitent des lignes dans l'Etat, le réseau total ouvre $2887 \mathrm{~km}^{1}$. Les lignes sont en relation directe avec la population et elles ne forment un réseau dense que dans le sud-est, entre Milwaukee, Portage, Madison et la frontière de l'Illinois. Des lignes atteignent les autres villes: Fond du Lac (à cette époque deuxième ville du Wisconsin par la population), Oshkosh, Appleton, Fort Howard (qui forme une agglomération avec Green Bay, de l'autre côté du Fox). Dans le centre, des lignes vont jusqu'à Berlin et Winneconne, points de départ de services de navigation fluviale. Deux lignes aboutissent au Mississippi et relient ainsi le lac Michigan et l'Etat de Minnesota; une troisième ligne, passant par.Eau Claire, ira jusqu'à Saint-Paul, mais elle s'arrête pour le moment à Menomonie. Dans le sud-ouest, une ligne venant de l'Illinois, dessert la région des mines. Une seule des seize villes du Wisconsin n'a pas encore de chemin de fer: Manitowoc, port sur le lac Michigan qui de ce fait n'est pas isolé. La plupart des localités de plus de mille habitants sont soit sur des voies ferrées soit à proximité de gares soit encore situées sur des voies d'eau navigables. Le reste du pays est très peuplé et ne compte aucune ville ou localité importante.

De 1870 à 1900 , l'immigration bat son plein dans le Wisconsin, dont la population passe de 1054670 à 2069 042; la ville de Milwaukee atteint 285315 habitants, tandis que Madison n'en a encore que 19 164. C'est aussi la grande période de construction des chemins de fer, mode de transport public qui

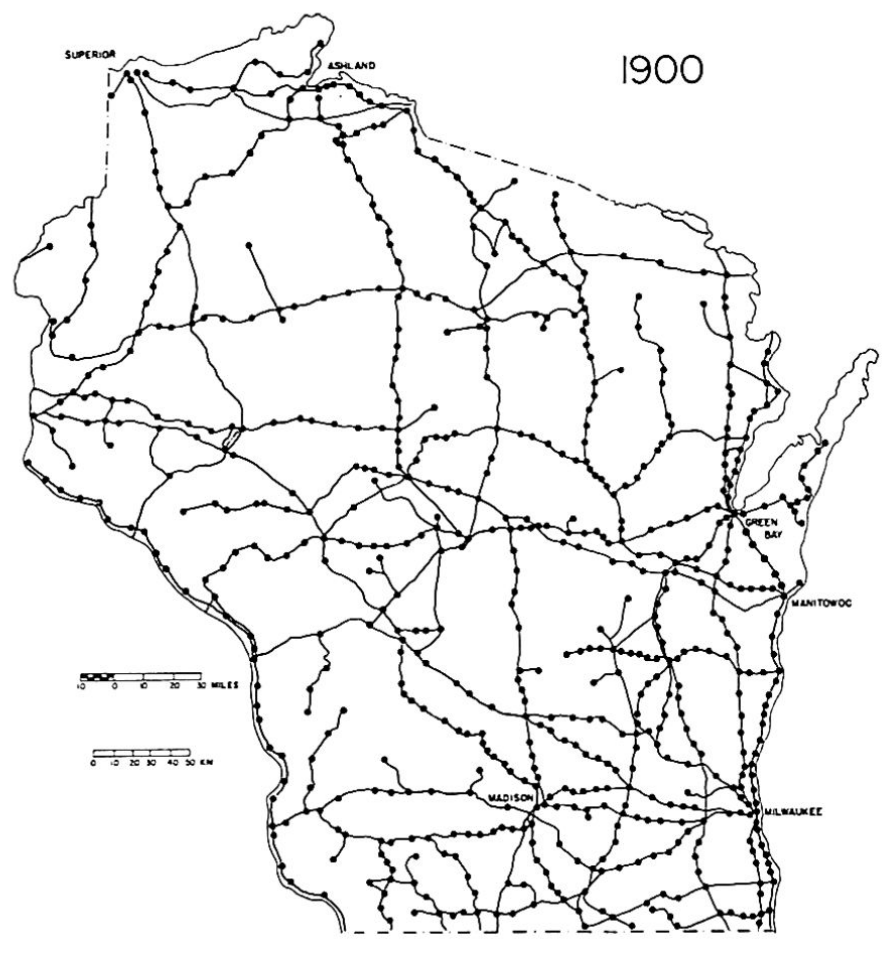

Figure 2. Lignes avec services de voyageurs en 1900

n'a pas de rival. Chicago est maintenant le centre ferroviaire incontesté du pays. Saint-Paul et la ville jumelle de Minneapolis constituent un autre nœud ferroviaire important. Milwaukee reste un point de convergence de lignes2, mais les grandes lignes ont leur point de départ à Chicago. La "Milwaukee, Saint-Paul Railway Company» est devenue la "Chicago, Milwaukee, Saint-Paul and Pacific Railroad Company», connue sous le nom de "Milwaukee Road». La concurrence s'est intensifiée, et les compagnies importantes luttent entre elles pour obtenir leur part du trafic entre les villes. Il y a maintenant quatre lignes entre Chicago et Saint-Paul, dont deux passent par Milwaukee, la "Chicago and North Western" et la "Milwaukee Road». Aux abords du Lac Supérieur, dans le Wisconsin, le Minnesota et le Michigan, l'exploitation de mines de fer a débuté et plusieurs lignes relient cette zone avec le Sud du Wisconsin et Chicago. Les ports d'Ashland et de Superior sont des points de convergence de voies ferrées. Toutes les villes et presque toutes les localités importantes sont maintenant desservies par le chemin de fer. Des lignes longent les voies navigables, montrant que la navigation n'a plus grande importance pour les relations à l'intérieur de l'Etat. Les lignes existantes ont poussé au développement des territoires qu'elles desservaient, et les seules régions sans voies ferrées sont les zones offrant peu de ressources. En 1900, il y a vingt-six compagnies de chemins de fer et $10509 \mathrm{~km}$ de lignes. Ce chiffre va encore augmenter sensiblement jusqu'à 1911, en particulier par la construction 


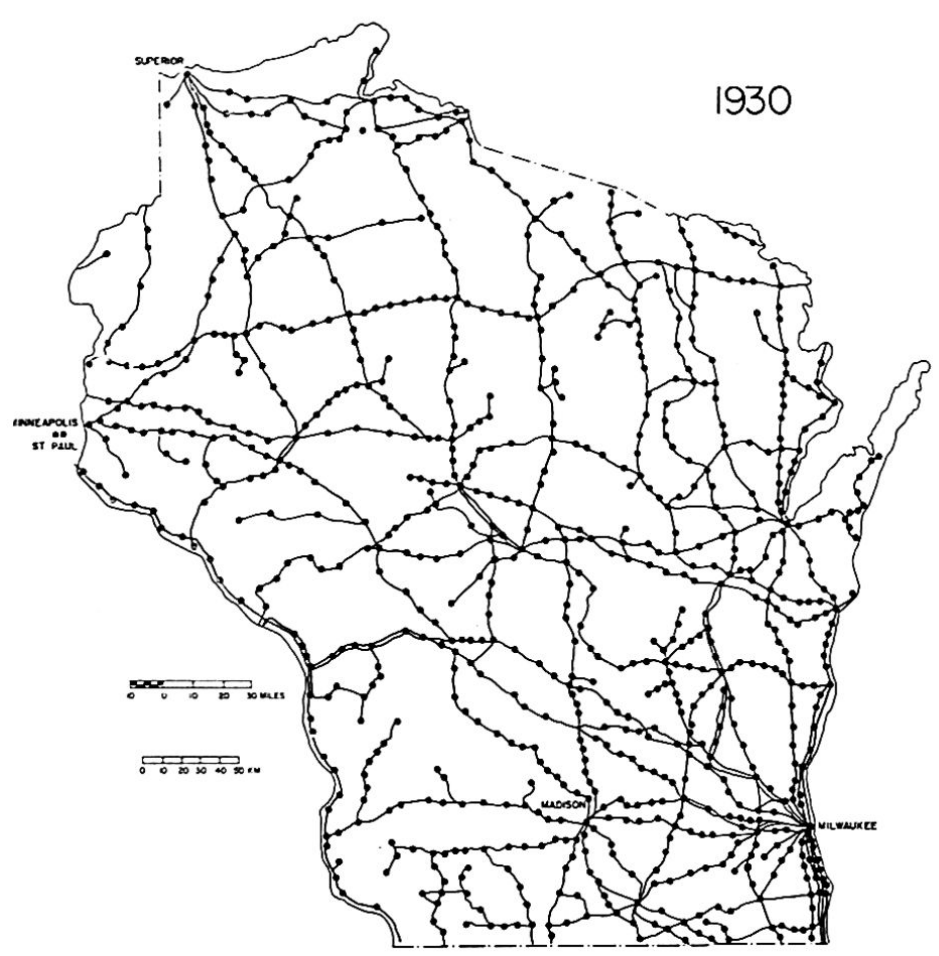

Figure 3. Lignes avec services de voyageurs en 1930

d'une ligne traversant l'Etat en diagonale de Milwaukee à Superior. Il semble cependant que les chemins de fer aient atteint le point de saturation. L'année 1920 marque l'extension maximale du réseau ferroviaire: $12382 \mathrm{~km}$. Dès 1872 , des lignes ont été abandonnées, mais il s'agit avant tout de tronçons peu importants, lignes rendues inutiles par l'établissement d'un raccordement plus avantageux ou lignes en relation avec des exploitations forestières ou minières abandonnées. Dès le début du siècle, cependant, les chemins de fer ont un rival, les transports automobiles. Assez rapidement l'automobile a acquis les faveurs de la population, surtout dans les campagnes et les villes petites ou moyennes. Les compagnies de chemins de fer réduisent le nombre de trains, suppriment les services de voyageurs sur certaines lignes, ou en abandonnent d'autres. Ces changements ne peuvent se faire qu'après une longue procédure, notamment une enquête qui doit établir que les services ne sont pas rentables et que les intérêts publics ne sont pas suffisants pour justifier leur maintien. De ce fait, en 1930 , il y a encore des services de voyageurs sur la plupart des lignes, et le réseau est encore assez près de sa plus grande extension ${ }^{3}$. Les principales lignes se répartissent entre quelques grandes compagnies: la "Chicago and North Western", la "Milwaukee Road", déjà notées en 1900, et la "Minneapolis, Saint-Paul et Sault-Sainte-Marie Railroad Company», connue sous le nom de "Soo Line».

De 1930 à 1965 , on assiste au déclin des services de voyageurs. Pendant un certain temps, les chemins de

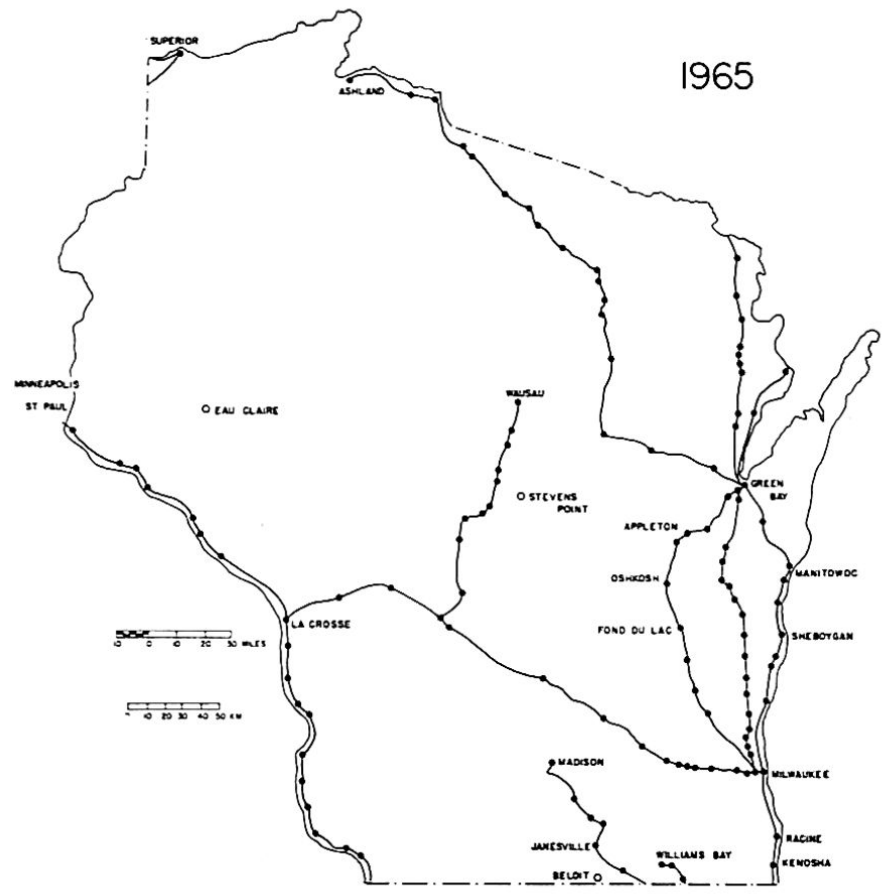

Figure 4. Lignes avec services de voyageurs en 1965

fer ont cherché à attirer la clientèle en améliorant les services (confort des wagons, wagons-restaurants, wagons-lits, bars, wagons avec dôme d'observation - compartiment surélevé dans une coupole entièrement vitrée, trains rapides), mais assez vite certaines compagnies se désintéressent des voyageurs, perdant ainsi leur clientèle et étant mieux en mesure de demander la suppression des trains. Au 31 décembre 1965, il ne reste plus dans le Wisconsin que cinq compagnies ayant encore des trains de voyageurs 4 . Deux d'entre elles, la «Northern Pacific Railway Company» et la "Great Northern Railway» n'exploitent, dans le Wisconsin, que des lignes entre Superior et le Minnesota. La "Chicago, Burlington and Quincy Railroad Company» exploite une ligne de Chicago à Saint-Paul qui, dans le Wisconsin, longe le Mississippi. La "Milwaukee Road» a également une ligne de Chicago à Saint-Paul; celle-ci passe par Milwaukee, elle a un embranchement qui va de Milwaukee à Green Bay et à Calumet dans le Nord du Michigan, un autre embranchement mène de New Lisbon à Wausau, enfin une ligne de la même compagnie relie Madison à Chicago, c'est la seule ligne desservant la capitale, cette dernière n'a plus de relations ferroviaires avec Milwaukee ou d'autres villes du Wisconsin. La "Chicago and North Western» exploite une ligne entre Chicago et Milwaukee, deux lignes entre Milwaukee et Green Bay, l'une par Sheboygan et Manitowoc, l'autre par Fond du Lac, Oshkosh et Appleton, une ligne de Green Bay à Ashland et une autre de Green Bay à Ishpeming dans le Michigan, enfin dans le 
Sud, une ligne de la banlieue de Chicago qui atteint le lac de Genève (Lake Geneva) ${ }^{5}$. Quelques services d'autocars dépendent de compagnies de chemins de fer, ils remplacent des services ferroviaires supprimés, notamment entre Madison et Columbus, sur la ligne de Milwaukee à Saint-Paul, et entre La Crosse, Eau Claire et Superior. Il existe en outre quelques lignes indépendantes d'autocars, notamment la compagnie Greyhound, elles relient entre elles les principales villes; la plupart de celles-ci ont un aérodrome commercial et sont desservies par des lignes aériennes. Quelques chiffres suffisent pour montrer clairement le déclin des services de voyageurs sur les lignes de chemins de fer du Wisconsin. C'est ainsi qu'en 1920, Madison avait trois gares, une par compagnie: la "Chicago and North Western» comptait dix-sept départs de trains par jour, en semaine, et quatorze le dimanche, l' 'Illinois Central» deux départs en semaine et un le dimanche, la "Milwaukee Road" quatorze en semaine et trois le dimanche6. C'était à peu près le même nombre de trains qu'en 1900 et en 1930. Par contre, en 1965, il n'y a plus que la gare de la "Milwaukee Road» qui offre un service de voyageurs et il n'y a plus que deux trains par jour. Stevens Point, petite ville de 18000 habitants, située au centre de l'Etat, à $150 \mathrm{~km}$ de la capitale et $300 \mathrm{~km}$ de Milwaukee, avait deux gares. En 1900, il y avait dix-huit départs de trains en semaine et six le dimanche, et l'on pouvait se rendre sans descendre de wagon jusqu'à Chicago, Milwaukee, Green Bay, Portage, La Crosse et Minneapolis. En 1920, il y avait vingt-trois départs en semaine et quatorze le dimanche; en plus des destinations indiquées ci-dessus, l'on pouvait aller directement jusqu'à Duluth. Aujourd'hui, il n'y a plus un seul train de voyageurs passant par cette ville.

Les trains n'ont été que partiellement remplacés par les services publics d'autocars ou d'avions. A Milwaukee et Madison, les relations avec d'autres métropoles se sont développées. Il y a actuellement plus d'une trentaine de vols journaliers entre Milwaukee et Chicago, une quinzaine entre Madison et Chicago, un autocar chaque demi-heure dans la journée entre Milwaukee et Chicago. Les relations des petites villes et des villages, par contre, ont sensiblement diminué. A Stevens Point, il y a actuellement sept départs d'avions par jour qui permettent d'atteindre directement Madison, Milwaukee, Chicago, Duluth et Minneapolis; il y a en outre une douzaine de départs quotidiens d'autocars à destination de Madison, Milwaukee, Chicago, Green Bay, Duluth, mais les horaires sont tels qu'il n'est pas possible d'aller dans les villes voisines d'Eau Claire ou de Wausau et d'en revenir le même jour. Quant aux petites localités, elles n'ont de transports publics que si elles sont situées sur une ligne d'autocar entre des villes ou sur une ligne de chemin de fer, mais la majorité des villages n'ont rien. Les habitants qui n'ont pas d'automobiles doivent faire appel à des taxis s'ils veulent se déplacer. Les principales voies ferrées existent encore, mais elles ne sont plus utilisées que pour le service des marchandises. Deux des compagnies actuelles ont un réseau étendu, mais sans service de voyageur: la "Soo Line» qui exploite des lignes de Chicago à Saint-Paul, de Chicago à Duluth et de Saint-Paul à Sault-Sainte-Marie, en traversant le Wisconsin, et la "Green Bay and Western» qui exploite une ligne de Green Bay, port sur le lac Michigan, et le Mississippi. Les autres compagnies n'ont que des réseaux de peu d'extention. Actuellement dans le Wisconsin, le nombre des voyageurs n'est pas élevé; en temps ordinaire, il n'y a plus guère dans les trains que des personnes âgées, des dames voyageant seules ou avec des enfants, des marins et des soldats. Il semble cependant qu'il y ait assez de voyageurs pour justifier le maintien des trains entre Chicago, Milwaukee et Saint-Paul et entre Milwaukee et Green Bay. La "Milwaukee Road» a manifesté l'intention de maintenir les services de voyageurs en construisant récemment une nouvelle gare à Milwaukee, gare toute moderne qui est même munie d'un passage sous-voie, le premier dans une gare du Wisconsin. Cette gare est utilisée aussi par les trains de la "Chicago and North Western". Les deux compagnies aimeraient fusionner, mais il n'est pas certain qu'elles puissent le faire, vu l'opposition d'autres compagnies. Il semble cependant que les embranchements de Green Bay à Calumet, à Ishpeming et à Ashland et celui de New Lisbon à Ashland ne subsisteront pas, car ils n'ont plus chacun qu'un train par jour qui transporte rarement plus d'une vingtaine de voyageurs à la fois. Si les chemins de fer ont été supplantés par les transports routiers et aériens, c'est que ceux-ci présentent beaucoup d'avantages. Pour les grandes distances, les avions sont rapides, pour les distances moyennes et courtes, les automobiles et les camions sont beaucoup plus avantageux, car véhicules et essence sont moins chers qu'en Europe, d'autre part, ils permettent des transports de porte à porte, sans intermédiaire et sans horaire fixe, ce qui est tout spécialement utile ici, car dans les banlieues des grandes villes, dans les petites villes et dans les villages, les habitations sont généralement des maisons familiales bâties au milieu d'un terrain plus ou moins étendu, ainsi les agglomérations s'étalent sur de vastes espaces et il n'est pas possible d'établir de services publics de transports d'un usage général. D'autre part, les chemins de fer n'ont jamais, dans le Wisconsin, joué le rôle qu'ils ont en Suisse ou dans la plupart des pays d'Europe. En dehors de l'agglomération de Milwaukee et de la région entre Milwaukee et Chicago, la population est en général peu dense, ainsi les trains ont rarement été utilisés par des ouvriers se rendant à leur travail, des enfants fréquentant les écoles, des ména- 
gères allant faire des emplettes ou des visites en ville. Même à Milwaukee, il n'y a jamais eu de service régulier de banlieue. Les écoles, les sociétés n'ont pas l'habitude de faire des courses et, dans le Wisconsin, le tourisme ne s'est développé qu'avec l'automobile. Il n'est donc pas étonnant que les chemins de fer aient vu le nombre de voyageurs décliner ce qui leur a permis de réduire les services. Lorsqu'il fut question de supprimer le dernier train desservant Stevens Point, l'enquête montra qu'en 1963, pendant toute l'année, il n'y avait eu que 522 voyageurs payants montés dans le train à cette gare.

La situation des chemins de fer dans le Wisconsin n'est pas unique, elle reflète la situation dans l'ensemble du pays, mais tout particulièrement la situation dans le centre et l'ouest. Dans l'est, où les villes sont nombreuses, le réseau ferroviaire reste dense, mais même là, le nombre des trains a sensiblement diminué. Avec l'augmentation de la population, la circulation routière devient de plus en plus difficile, et il est maintenant question d'établir de nouveaux services ferroviaires rapides entre les grandes villes de la côte est. Il est possible que dans un avenir plus ou moins éloigné, les trains regagnent de l'importance.

\section{Bibliographie}

The Official Guide of the Railways and Steam Navigation Lines of the United States, Porto Rico, Canada, Mexico and Cuba, dès 1871 (le titre a varié).

The Blue Book for the State of Wisconsin, dès 1879 . (Publication officielle contenant les rapports d'activité du gouvernement et des agences gouvernementales.)

Thwaites, Reuben G.: The Story of Wisconsin. Boston 1891.

The Railroads of Wisconsin, 1827-1937. Boston 1937.

Stover, John F.: American Railroads. Chicago 1961.

Les publications sur les chemins de fer sont très nombreuses, voir: Bibliography of railway literature, $6^{\mathrm{e}}$ édition. Washington 1957.

\section{Note}

MM. Raymond E. Specht et Maurice E. Perret sont tous deux professeurs de géographie à l'Université d'Etat du Wisconsin à Stevens Point. En 1964 et
1965 , ils ont reçu une subvention du Fonds de recherche de l'Université pour faire une étude sur les chemins de fer dans l'Etat. M. Specht a étudié plus spécialement la question des transports de marchandises, tandis que M. Perret s'intéressait aux services de voyageurs. Les premiers résultats de cette étude ont paru dans le "Bulletin of the Wisconsin Council for Geographic Education», printemps 1966, sous le titre "Geographical Analysis of Wisconsin Railroads (A report of some preliminary findings)».

\section{Zusammenfassung}

Die erste Eisenbahnlinie im amerikanischen Staate Wisconsin geht auf das Jahr 1850 zurück. Sie sollte im Endausbau der Verbindung wichtiger Schiffahrtswege, des Michigansees und des Mississippi, dienen; zunächst handelt es sich indessen bloß um eine Strecke von $16 \mathrm{~km}$, zwischen Milwaukee und Wauwatosa. Verschiedene private Gesellschaften wetteifern in der Folge um die Entwicklung des Bahnnetzes, dessen Länge bereits 1856, als die Verbindung zum Mississippi vollendet war, $816 \mathrm{~km}$ betrug und sich bis 1920 auf volle $12382 \mathrm{~km}$ ausweitete. Die Konkurrenz durch die neu aufkommenden Verkehrsmittel auf der Straße und in der Luft setzte dem Ausbau dann freilich eine Grenze, und der Niedergang des Bahnwesens ließ nicht auf sich warten, zumal die Aufmerksamkeit der Gesellschaften dem Personenverkehr gegenüber bald einmal zu wünschen übrig ließ. Der Aufsatz schildert abschließend die heutige Situation der Eisenbahnen von Wisconsin, welche die in den zentralen und westlichen Teilen der USA vorliegenden Verhältnisse im ganzen getreulich widerspiegelt.

\section{Remarques}

1 Voir figure 1.

2 Voir figure 2.

3 Voir figure 3.

4 Voir figure 4.

5 En 1965 , le terminus de cette ligne était Williams Bay. En 1966, l'exploitation a cessé entre Williams Bay et la ville de Lake Geneva qui est ainsi devenu le terminus.

6 A l'instar de l'Angleterre, certaines compagnies américaines n'avaient que des services réduits le dimanche par respect pour le jour du repos. 